\section{Apuntes sobre la historia de la cirugía cardiaca en Costa Rica}

\author{
Notes on the history of cardiac surgery in Costa Rica
}

Dr. Rodrigo Gutiérrez-Aguilar

\section{Resumen}

La historia de la cirugía cardiaca en Costa Rica, señala tres fechas fundamentales de las que por azares del destino he sido testigo directo o indirecto.

1 de Mayo de 1917. Primera sutura de una herida del corazón en Costa Rica.

El 9 de setiembre de 1896 el alemán Ludwig Rehn, sutura con éxito una herida del corazón de un jardinero de 22 años, esa fecha es reconocida en todo el mundo como el inicio de la cirugía cardiaca, acabando así con el mito de la inviolabilidad quirúrgica de ese órgano y borrando la frase del influyente cirujano Theodore Billroth: "El cirujano que trate de suturar una herida del corazón, perderá el respeto de sus colegas".

Doce años después, el joven alajuelense Julio Aguilar Soto se graduaba como médico en la prestigiosa universidad de Tulane en Louisiana, habiendo recibido entrenamiento en cirugía de torax con el Dr. Rudolph Matas, histórico pionero en ese campo. Pronto formaría parte de un distinguido grupo de cirujanos que hacían verdaderos prodigios en el Hospital San Juan de Dios, como el que ocurrió el primero de mayo de 1917 y al que el Dr. Aguilar Soto evitaba referirse, de acuerdo a su personalidad de sólidos conocimientos científicos, pero ajeno a los elogios y reconocimientos externos, soy testigo de ello, ya que gracias a un parentesco familiar lo pude conocer y departir con él varias veces allá por los años 60 .

\section{Abstract}

The history of cardiac surgery in Costa Rica has three fundamental dates, which I have been able to witness due to a twist of fate.

1 May 1917. First suture of a heart wound in Costa Rica.

On 9 September 1896 the German, Ludwig Rehn, performed a successful suture of a heart wound in a 22-year old gardener. This date is recognized worldwide as the inauguration of heart surgery, thus ending the myth of surgical inviolability of such organ and erasing the announcement made by the influential surgeon Theodore Billroth, "The surgeon who should attempt to suture a wound of the heart would lose the respect of his colleagues."

Twelve years later, the young Julio Aguilar Soto, from the province of Alajuela, graduated as a physician from prestigious Tulane University in Louisiana, after training in chest surgery with Rudolph Matas, a pioneer in this field. He would soon form part of a distinguished group of surgeons who performed true wonders at the San Juan de Dios Hospital, such as the one concluded on 1 May 1917. Dr. Aguilar Soto avoided talking about this case, due to his reluctance to being praised, despite his solid scientific knowledge. I witnessed this because we had family ties, thus I got to know and share with him a few times back in the 60s.

Descriptores: historia, cirugía cardiaca, Costa Rica

Keywords: history, cardiac surgery, Costa Rica 
La historia de la cirugía cardiaca en Costa Rica, señala 3 fechas fundamentales de las que, por azares del destino, he sido testigo directo o indirecto.

\section{$1^{\circ}$ de mayo de 1917 . Primera sutura de una herida del corazón en Costa Rica}

El 9 de septiembre de 1896, el alemán Ludwig Rehn, sutura con éxito una herida del corazón de un jardinero de 22 años; esa fecha es reconocida en todo el mundo como el inicio de la cirugía cardiaca, acabando así con el mito de la inviolabilidad quirúrgica de ese órgano, y borrando la frase del influyente cirujano Theodore Billroth: "El cirujano que trate de suturar una herida del corazón, perderá el respeto de sus colegas”.

Doce años después, el joven alajuelense Julio Aguilar Soto, se graduaba como médico en la prestigiosa universidad de Tulane en Louisiana, habiendo recibido entrenamiento en cirugía de tórax con el Dr. Rudolph Matas, histórico pionero en ese campo. Pronto formaría parte de un distinguido grupo de cirujanos que hacían verdaderos prodigios en el Hospital San Juan de Dios, como el que ocurrió el $1^{\circ}$ de mayo de 1917, y al que el Dr. Aguilar Soto evitaba referirse, de acuerdo con su personalidad de sólidos conocimientos científicos, pero ajeno a los elogios y reconocimientos externos.

Soy testigo de ello, ya que gracias a un parentesco, pude conocerlo y departir con él varias veces, allá por los años 60 . De nuestras reuniones tengo imborrables recuerdos, pero 2 me acompañarán el resto de mi vida. Un día, al despedirnos me dijo: "Quiero regalarle mis libros de cirugía". A pesar de mi negativa inicial, hoy los guardo como un invaluable tesoro. El segundo recuerdo lo comparto ahora con ustedes, ya que ante mi insistencia, en una ocasión me comentó: "Bueno, voy a relatarle los detalles que recuerdo de aquel $1^{\circ}$ de mayo de 1927; ese día recibimos de emergencia a un muchacho de apellido Jaramillo, con una herida de puñal en el pecho. Venía muy mal, cianótico y disneico; creo que mi mayor mérito fue diagnosticar

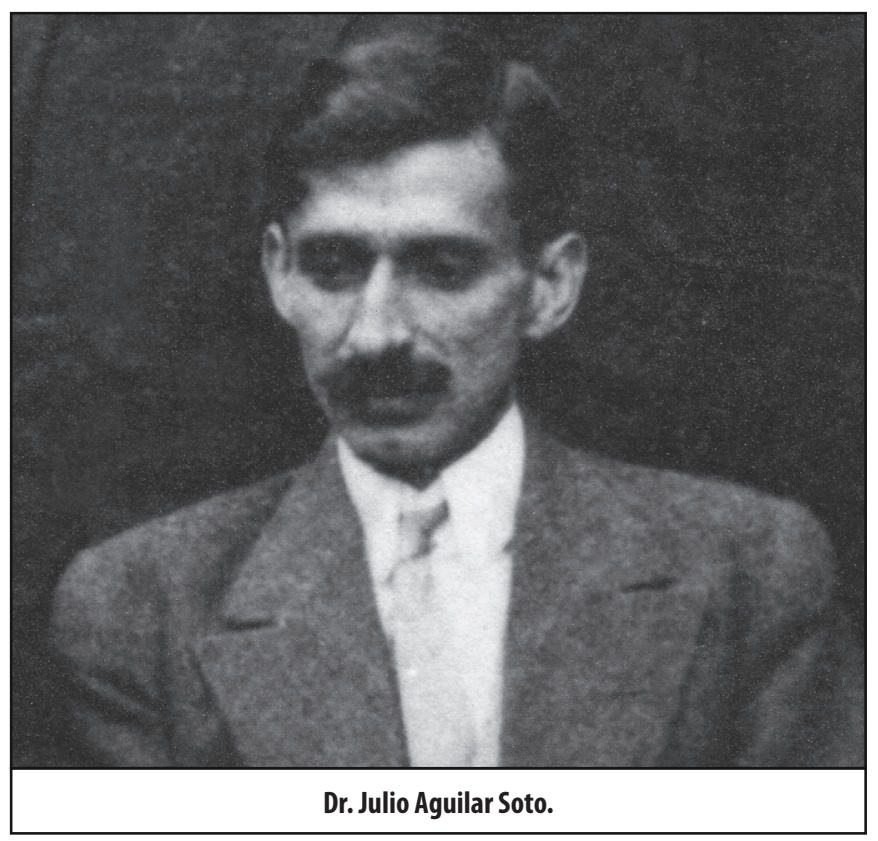

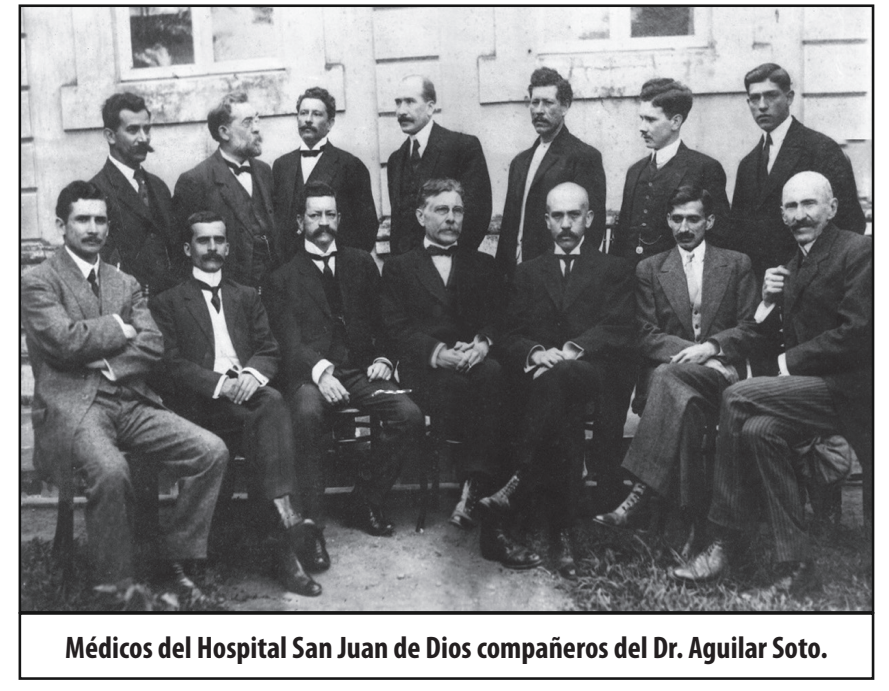

de inmediato la herida en el corazón. En ese entonces los cirujanos del San Juan nos alternábamos en equipos formados por el cirujano principal, un ayudante y un encargado de la anestesia, ya que no existía la especialidad de anestesiología, de modo que lo que hacíamos era turnarnos en esas funciones. Debo confesarle que mi grupo favorito era el que completaban el Dr. José María Soto Alfaro y el Dr. Vicente Castro Cervantes. Sin perder un segundo llevamos al paciente a la sala de operaciones, llegamos hasta el corazón y comprobamos mi diagnóstico, así que coloqué un dedo sobre la herida y pude controlar la hemorragia, pero teníamos el campo operatorio totalmente lleno de sangre, y decidimos inclinarlo lateralmente para evacuarla".

Interrumpo las palabras del Dr. Aguilar para hacer un paréntesis dedicado a los actuales cirujanos torácicos: por un momento piensen en una situación como la descrita, sin tener succión convencional ni, mucho menos, sistemas de autotransfusión o salvador de células, lo que magnifica la heroicidad de la maniobra.

Y sigo con la narración del Dr. Aguilar. "Sin quitar el dedo de la herida, coloqué dos puntos de sutura y pude comprobar que ya no sangraba, sin embargo, a pesar de nuestro esfuerzo, el paciente falleció al día siguiente".

La operación del Dr. Aguilar Soto, marca el inicio de la cirugía cardiaca en Costa Rica.

\section{$1^{\circ}$ de mayo de 1963. Primera operación bajo circulación extracorpórea en Costa Rica.}

El 6 de mayo de 1953, el doctor John H. Gibbon Jr., utilizó con éxito un sistema corazón-pulmón artificial, para corregir una comunicación interauricular en una muchacha de 18 años, naciendo así la cirugía a corazón abierto bajo circulación extracorpórea. Pero, en el mismo año el Dr. Gibbon decidió suspender ese procedimiento y regresar al laboratorio experimental, debido a 2 fracasos después de su éxito primero. El problema fundamental era lo complejo de su sistema extracorpóreo, basado en múltiples membranas de difícil manejo. Para algunos todo había sido un sueño, una quimera. A pesar de eso, en 1955 se produce un cambio total, cuando el Dr. 


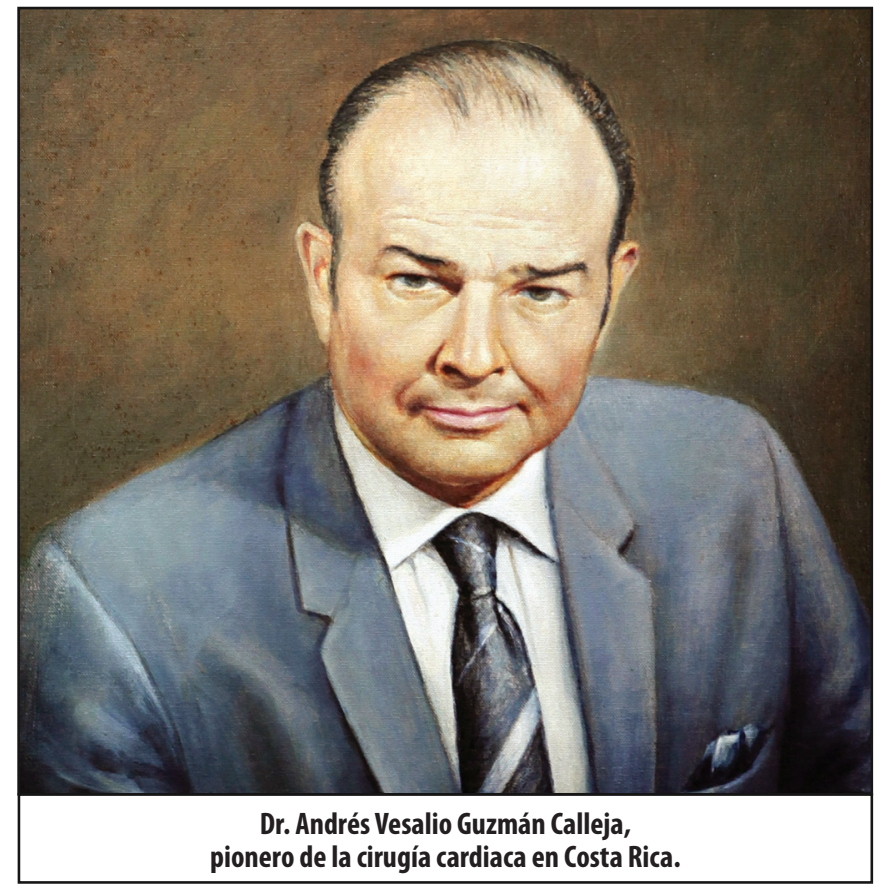

Richard De Wall opera con éxito a 7 niños, utilizando su propio diseño de un oxigenador de burbujas, y haciendo renacer la esperanza de los cirujanos de corazón. En Costa Rica, el Dr. Andrés Vesalio Guzmán no solo estaba convencido que este era el futuro de la cirugía cardiaca, sino que decidió construir su propio oxigenador y después de arduos y bien planificados años de estudios experimentales, lo logra.

Mi amistad con el Dr. Guzmán se origina en el arraigo cartaginés de las familias Guzmán Calleja y la mía, lo que me permitió vivir con intensidad aquellos años de investigación en el laboratorio experimental del Hospital San Juan de Dios.

En un momento culminante, obtuvimos el primer éxito con la sobrevida de un perro llamado "Capitán". El Dr. Guzmán organizó de inmediato una fiesta de celebración con la presencia de "Capitán" y, como principal invitado, el Dr. José Manuel Quirce Morales, director del Hospital. En determinado momento, entre tazas de café, refrescos y bocadillos, se presentó el siguiente diálogo.

-Bueno, José Manuel, ya estamos listos para hacer este procedimiento en el humano, ¿Voz nos darías permiso? -dijo el Dr. Guzmán con voz muy fuerte.

- Sí, sí, como no, - contestó entre risas el Dr. Quirce.

- Muchachos, ya tenemos el permiso.- dijo Vesalio, casi en susurro.

La operación se organizó con base en 3 puntos fundamentales: 1- La paciente sería Rosa Montero, de 17 años de edad, quien presentaba una estenosis de la válvula pulmonar. 2- Solo el grupo que iba a participar lo sabría. 3- Se llevaría a cabo el $1^{\circ}$ de mayo, día feriado, con el mínimo del personal trabajando. Yo sugerí que filmáramos el acontecimiento con una cámara de cine propiedad de mi padre, y así se hizo. Todo se desarrolló de maravilla, pero para que apareciera la piedra en el zapato, algún empleado despistado y chismoso, con la operación avanzada, corrió a llamar al Dr. Quirce para alertarlo sobre algo irregular que estaba sucediendo en la sala de operaciones. En el momento cuando se terminaba de cerrar el tórax de la paciente, apareció el señor director en la puerta de la sala y casi gritando dijo:

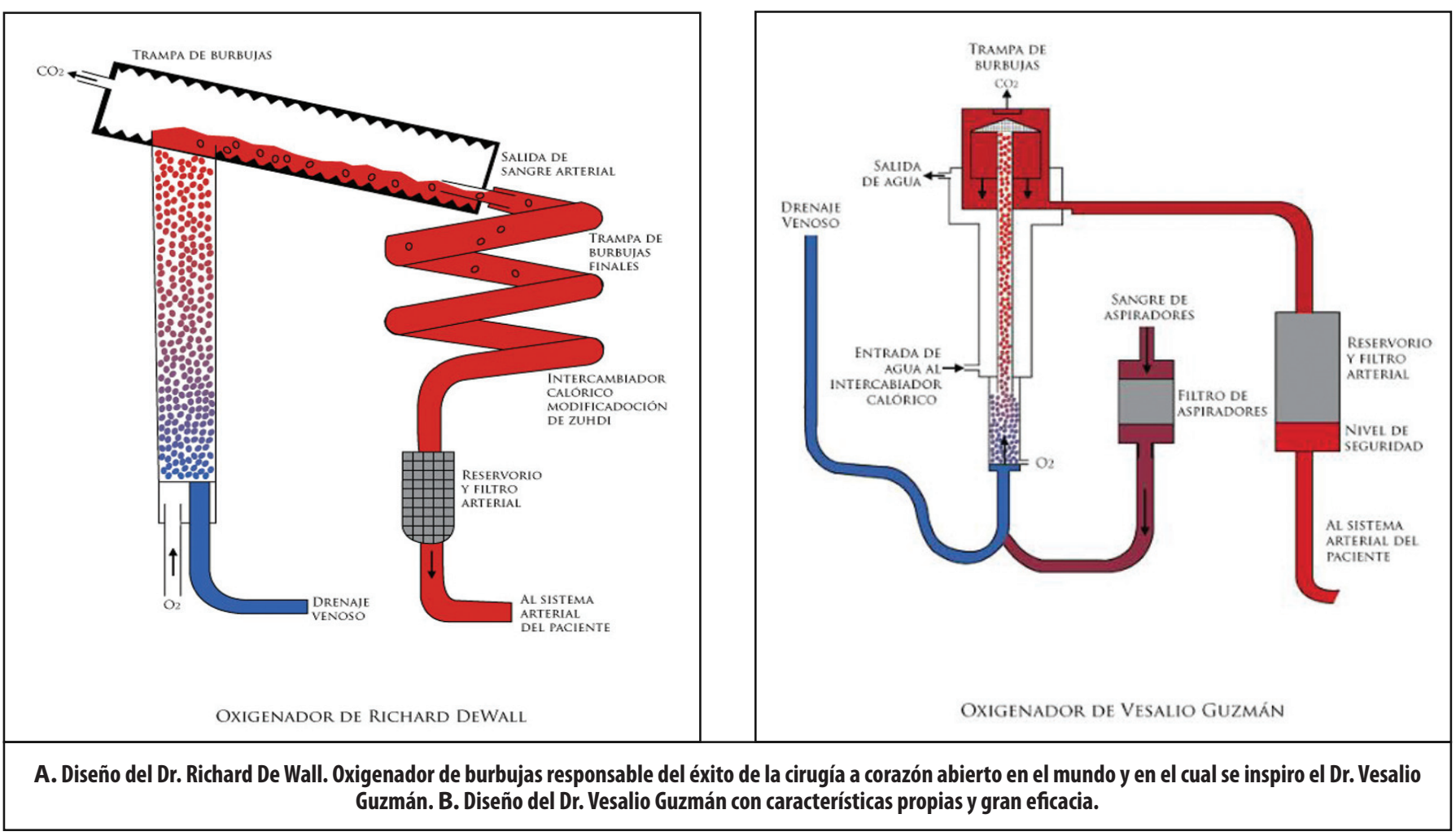




\section{-¿Qué pasa aquí?}

-Vos nos diste el permiso y aquí está la primera cirugía a corazón abierto que se hace en Costa Rica, y con éxito total.-Contestó el Dr. Guzmán, con toda parsimonia.

11 años antes, el 27 de mayo de 1952, el Dr. Vesalio Guzmán había realizado la primera comisurotomía mitral a corazón cerrado, y por solo este hecho debe ser recordado como el pionero de la cirugía cardiaca en el país, pero la magnitud de los acontecimientos que culminaron con éxito en mayo del 63, merecen resaltarse en letras de oro. El Dr. Guzmán decía que se había inspirado en el modelo de De Wall para construir su oxigenador, pero la verdad es que su diseño tiene características que le dieron identidad: más compacto, intercambiador calórico incorporado, efectivo atrapador de burbujas, etc., según es posible observar en los esquemas adjuntos. Construido totalmente en Costa Rica, se utilizó con éxito en muchos pacientes.

Con el paso de los años evolucionaron los oxigenadores, hasta llegar a los de membrana de fibra capilar, pero sin duda alguna aquellos primeros de burbujas, fueron determinantes en la cirugía cardiaca. Aparte del Dr. De Wall, la historia señala diseños de ilustres cirujanos: Clark, Jordan, Rygg, Cooley, Zuhdi y muchos más, pero en Costa Rica se debe recordar con orgullo el oxigenador del Dr. Guzmán, responsable del inicio de la cirugía a corazón abierto en nuestro medio.

\section{8 de marzo de 1991, primer trasplante de corazón en Costa Rica}

El 3 de diciembre de 1967, el Dr. Christiaan Barnard, en Sudáfrica, realiza el primer trasplante cardiaco, al comerciante de ultramarinos Louis Washkanski. En ese mismo año, en el Hospital Central de la CCSS, hoy Hospital Dr. Calderón Guardia, el Dr. Longino Soto Pacheco trataba de crear un servicio exclusivo para la cirugía cardiaca, la cual se realizaba como un agregado en los servicios de cirugía general, tanto en ese hospital como en el San Juan De Dios. El momento era propicio, ya que estábamos a punto de estrenar el nuevo Hospital México, y la hazaña del Dr. Barnard fortaleció aun más la necesidad del futuro servicio. Reorganizamos entonces el laboratorio de cirugía experimental, dándole énfasis al trasplante cardiaco. Fueron meses de mucho trabajo, llenos de ilusión y mística, soñando con el futuro servicio de cirugía cardiaca, y en el fondo casi en forma latente, convencidos de que un trasplante cardiaco en nuestro medio era algo utópico, ya que después del caso de Barnard, solo se realizaba en unos pocos hospitales en el mundo, y con poco éxito. A partir de 1980, el panorama de los trasplantes cardiacos cambió en forma radical, debido a 3 factores: los inmunosupresores, la biopsia endocárdica y la red de disponibilidad de donadores.

Para 1991 el proyecto del servicio de cirugía cardiaca en el Hospital México era una realidad, pero el Dr. Longino Soto, vehemente y polémico, tanto en el deporte como en la política o la medicina, tenía muchos amigos y también un buen grupo de enemigos. Al cumplir 67 años, la Junta Directiva de la CCSS decidió jubilarlo en forma obligada, y a pesar de la

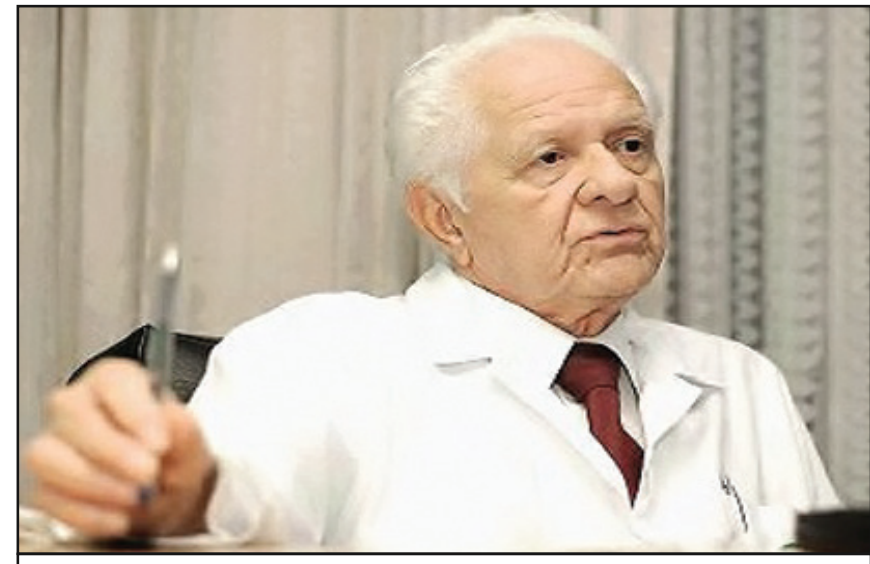

Dr. Longino Soto Pacheco. Primer trasplante de corazón en Costa Rica.

oposición acalorada del Dr. Soto, solo una apelación de otro médico detenía la decisión de las autoridades de la Caja. En el momento álgido de esta controversia, ingresa al Hospital el guanacasteco Juan Rueda, cuya única posibilidad de sobrevida era un trasplante cardiaco, y el Dr. Soto no lo duda y de inmediato inicia los preparativos para la operación, pero con 2 grandes interrogantes: la disponibilidad de un donador y los controversiales aspectos legales del procedimiento, que hacían dudoso el permiso de la dirección del hospital. El 6 de marzo, otro guanacasteco de 17 años de edad, sufre una caída desde un camión de carga y llega al hospital con severas lesiones, las cuales le causan muerte neurológica; los estudios de laboratorio muestran que es en todo compatible con Juan Rueda, y la familia del joven accidentado da su consentimiento. Ante la falta de un pronunciamiento legal claro, el Dr. Soto me dijo:

-Macho, esto 10 manejamos como una emergencia médica; yo asumo toda la responsabilidad.

El procedimiento quirúrgico se inició a las 3:30 p.m., en forma acelerada, debido al paro cardiaco del donador. A las 7:45 p.m., la operación había terminado, pero faltaban los momentos de mayor angustia, ya que el nuevo corazón de Juan, prácticamente no se movía.

Christiaan Barnard había dicho después de su trasplante "la comprobación de que ante mí se hallaba tendido un hombre sin corazón pero vivo, me parece que fue el momento que me infundió más pavor". Pero en mi caso, el momento de gran ansiedad se produjo al comprobar la insuficiencia del nuevo corazón. El futuro físico de Juan Rueda y el profesional del Dr. Soto, dependían de los sistemas extracorpóreos que yo tenía en mis manos. Longino se quitó los guantes y la bata de operar, dio una vuelta por la sala, sin decir una sola palabra, puso una mano sobre mi hombro y se retiró; un cirujano asistente dijo "Por 10 menos hicimos el primer intento.". Cada minuto siguiente se hizo del tamaño de un siglo, las cifras del laboratorio clínico, encabezadas por un hematocrito de 15 , eran alarmantes.

Con dos intensivistas, el Dr. Oscar Palma y el Dr. Mauricio Artiñano, el soporte hemodinámico extracorpóreo y un hemoconcentrador, la mejoría del paciente fue sucediéndose minuto a minuto, por las siguientes largas 4 horas, hasta que 
a las 12:20 a.m., lo pudimos trasladar a la sala de cuidados intensivos.

Me retiré a dormir como a las 3:00 a.m. y a las 6:00 a.m. me despertó el timbre del teléfono; contesté esperando lo peor, pero era Longino, quien a viva voz decía: - "Macho... Juan está como si le hubiéramos sacado una uña del pie."

E1 Dr. Longino Soto continuó haciendo complicadas cirugías cardiacas por los siguientes 15 años, incluyendo 8 trasplantes cardiacos. Uno de esos, el practicado a Blanca Vega, está entrando en el récord mundial de sobrevida, tras 23 años de efectuado el trasplante.

Hoy, nuestra cirugía cardiaca está consolidada y modernizada con sistemas extracorpóreos digitalizados y computarizados, el cateterismo terapéutico, las técnicas mínimamente invasivas, la necesaria incorporación de la robótica, el futurista aporte de la nanocirugía, etc., pero rindamos tributo permanente a los pilares que establecieron las bases de lo que tenemos: Dr. Julio Aguilar Soto, Dr. Andrés Vesalio Guzmán Calleja, Dr. Longino Soto Pacheco, y en la cardiocirugía pediátrica, Dr. Roberto Ortiz Brenes.

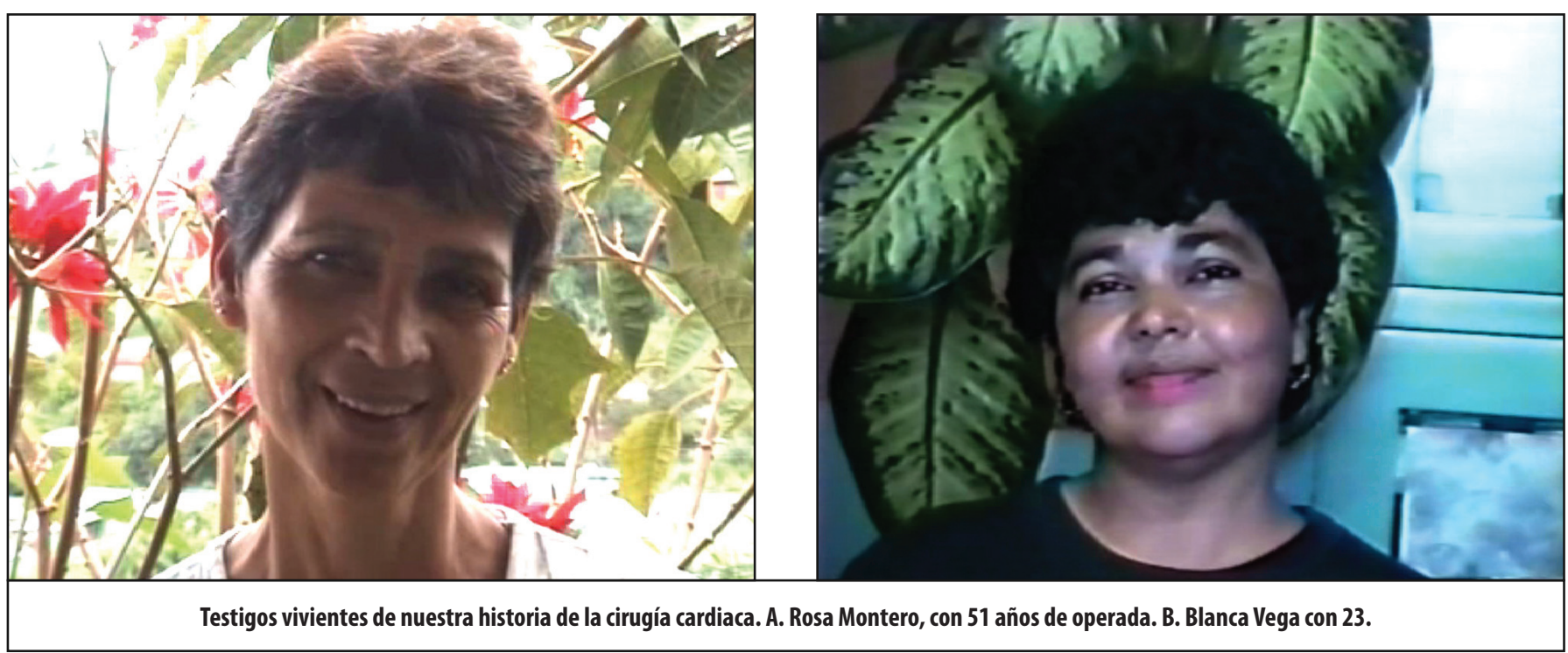

\footnotetext{
Fe de erratas

En la revista volumen 56, número 2 se debe corregir la siguiente información:

1. En el artículo Análisis de pacientes drepanocíticos tratados con hidroxiurea en el Hospital Nacional de Niños en página 51, columna derecha línea 4 el texto: "Cuadro 5" debe ser eliminado ya que no corresponde.

2. En página 58, la referencia número 10 se debe leer: "Clark WH Jr, From L, Bernardino EA, Mihm MC. The histogenesis and biologic behavior of primary human malignant melanomas of the skin. Cancer research 1969;29:705-726."

3. En página 58, la referencia número 11 se debe leer: "Azzola M, Shaw HM, Thompson JF, Soong SJ, Scolyer RA, Watson GF, et al. Tumor Mitotic Rate Is a More Powerful Prognostic Indicator than Ulceration in Patients with Primary Cutaneous Melanoma. Cancer 2003:97:1488-1498. "

4. En página 63, la referencia número 11 se debe leer: "Caglevic C, Anabalón J, Baeza R. Manejo del dolor oncológico en un hospital público de Santiago de Chile. Rev Chil Cancerol Hematol 2008;17:21-26."

5. En página 68, columna izquierda último párrafo se debe leer: (Cuadro 2).

6. En la página 77, la referencia 18 se debe leer: "18. Propper C, Sutton M, Whitnall C, Windmeijer F. Did «targets and terror» reduce waiting times in England for hospital care? B E J Econ Anal Pol 2008; 8:5."

7. En la página 77, la referencia 19 se debe leer: "Wilcox S, Seddon M, Dunn S, Edwards RT, Pearse J, Tu JV. Mesuring and reducing waiting times: a cross-national comparison of strategies. Health Aff (Millwood)2007; 26:1078-87."

8. En la página 77, la referencia 19 se debe leer la referencia 21 como 20, la 22 como 21, la 23 como 22, la 24 como 23 , la 25 como 24 y la 26 como 25.

9. Entre las páginas 74 y 76 , se deben ignorar los superíndices del 26 al 33, ya que no corresponden a referencias bibliográficas.
} 